9th A. Friedmann International Seminar and

3rd Casimir Symposium 2015

International Journal of Modern Physics: Conference Series

Vol. 41 (2016) 1660143 (6 pages)

(C) The Author(s)

DOI: $10.1142 /$ S2010194516601435

\title{
Simulation of laser ablation of metals for nanoparticles production
}

\author{
R. V. Davydov \\ Peter the Great St. Petersburg Polytechnic University \\ St. Petersburg, 195251, Russia \\ davydovroman@outlook.com \\ V. I. Antonov \\ Peter the Great St. Petersburg Polytechnic University \\ St. Petersburg, 195251, Russia \\ T. I. Davydova \\ Peter the Great St. Petersburg Polytechnic University \\ St. Petersburg, 195251, Russia \\ Received 17 September 2015 \\ Published 18 March 2016
}

\begin{abstract}
In this paper a mathematical model for femtosecond laser ablation of metals is proposed, based on standard two-temperature model connected with 1D hydrodynamic equations. Wide-range equation of state has been developed. The simulation results are compared with experimental data for aluminium and copper. A good agreement for both metals with numerical results and experiment shows that this model can be employed for choosing laser parameters to better accuracy in nanoparticles production by ablation of metals.
\end{abstract}

Keywords: Laser ablation; femtosecond lasers; nanoparticles; aluminium; copper.

PACS numbers: 47.85.Dh, 64.30.Ef, 79.20.Eb

\section{Introduction}

There is a growing interest in the nanofabrication of materials and their applications in various fields of life and technology, such as electronics, energy generation, health care and storage. A great deal of progress in this field has relied on the use of lasers. Production of nanoparticles can be done in several ways, one of them is laser ablation. ${ }^{1}$

This is an Open Access article published by World Scientific Publishing Company. It is distributed under the terms of the Creative Commons Attribution 4.0 (CC-BY) License. Further distribution of this work is permitted, provided the original work is properly cited. 
Despite extensive research work, accurate prediction of the ablation process is still lacking, because it significantly depending on laser parameters, surrounding medium and target material characteristics. To analyze the physical processes at high energy densities, when laser is used, an adequate description of the thermodynamic properties of matter over a broad region of states including the normal conditions and plasma at high pressures and temperatures is required.

Nowadays a two-temperature model (TTM) has been widely employed for solving ultrashort laser processing of metals. ${ }^{2-5}$ This continuous model describes the energy transfer inside a metal with two coupled generalized heat conduction equations for the temperatures of the electrons and the lattice. To describe the material removal processes TTM is often inserted into a hydrodynamic code. But the choice of an equation of state, required for solving hydrodnamic equations, can significantly affect the results.

The article is organized as follows. It begins with describing the mathematical model, then we construct the appropriate semi-empirical two-temperature equation of state for model. Finally, we demonstrate the results for aluminium and copper and compare it with experimental data.

\section{Mathematical Model}

In this work we describe the evolution of material parameters using the conservation of mass, momentum and energy of electron, and ion subsystems in a twotemperature single-fluid 1D Lagrangian form:

$$
\begin{gathered}
\frac{\partial}{\partial t}\left(\frac{1}{\rho}\right)+\frac{\partial v}{\partial m}=0 \\
\frac{\partial v}{\partial t}+\frac{\partial P}{\partial m}=0, v=\frac{\partial x}{\partial t} \\
\frac{\partial \varepsilon_{e}}{\partial t}+P_{e} \frac{\partial v}{\partial m}=\frac{\partial}{\partial m}\left(k \rho \frac{\partial T_{e}}{\partial m}\right)-\frac{\alpha_{e i}}{\rho}\left(T_{e}-T_{i}\right)+J_{L}, \\
\frac{\partial \varepsilon_{i}}{\partial t}+P_{i} \frac{\partial v}{\partial m}=\frac{\alpha_{e i}}{\rho}\left(T_{e}-T_{i}\right) .
\end{gathered}
$$

Here, $m$ is the mass coordinate, $d m=\rho d x, m=\int_{x 0}^{x} \rho d x$, direction of $x$-axis is chosen perpendicular to the irradiated surface of the metal, $\rho$ is the density, $\rho_{0}$ is the initial density, $u$ is the velocity, $t$ is the time, $P_{e}$ and $P_{i}$ are the pressures of electrons and ions, $\varepsilon_{e}$ and $\varepsilon_{i}$ are the internal energies of electrons and ions, $P=P_{e}+P_{i}$ and $\varepsilon=\varepsilon_{e}+\varepsilon_{i}$ are the full pressure and the internal energy, $T_{e}$ and $T_{i}$ are the temperatures of electrons and ions.

For the coefficient of electron-ion relaxation $\alpha_{e i}$ we use formula from work Ref. 6 and energy of the absorbed laser radiation $J_{L}$, described by:

$$
J_{L}=\frac{F_{a b s}}{\tau_{L} \delta \sqrt{\pi} \rho} \exp \left(-\frac{t^{2}}{\tau_{L}^{2}}\right) \exp \left(-\frac{x(m, t)-x\left(m_{0}, t\right)}{\delta}\right) .
$$


Here $\tau_{L}$ is the laser pulse duration, $F_{a b s}$ is the laser radiation energy, $\delta$ is the skin depth of metal.

The electron wide-range thermal conductivity coefficient $k$ is constructed as an interpolation between limiting metal and hot plasma cases. ${ }^{7}$ The viscosity and thermal conductivity of the ions are neglected in this model. To calculate the ablation depth, we perform an integration of the mass flux through the surface using the expression:

$$
d=\left.\frac{1}{\rho_{0}} \int_{0}^{t}(\rho u)\right|_{x=x_{0}} d t^{\prime}
$$

\section{Equation of State}

To solve the system of hydrodynamic equations (1) - (4) we construct the widerange semi-empirical two-temperature equation of state. The metal is expected to consist of the same electrically neutral atomic cells with atomic weight $A$ and charge $Z$. The Helmholtz free energy of a single atomic cell is the sum of three terms, describing the electronic and ionic components, and the interaction between them:

$$
F=F_{e}+F_{i}+F_{e i}
$$

As the main thermodynamic parameters we use atomic cell volume $\mathrm{V}$ and temperatures $T_{e}, T_{i}$. The number of free electrons in one atomic cell we call the degree of ionization and denote by the letter $y$. The pressure of the electrons is assumed to be the pressure of an ideal Fermi gas of electrons with density $y / V$ and temperature $T_{e}$, where $\mathrm{y}$ is the solution of the modified equation of ionization:

$$
\mu_{F}\left(\frac{y}{V}, T_{e}\right)+I(y)-B\left(V, T_{e}\right)=0 .
$$

Here $\mu_{F}$ - the chemical potential of an ideal Fermi gas, $I(y)$ - ionization potential, which is a smooth function of $y$ and is constructed as a smoothing spline on the known experimental values of the successive stages of ionization $I(1), I(2)$ etc. $B\left(V, T_{e}\right)$ - negative correction, which purpose is the description of cold ionization of highly compressed matter:

$$
B\left(V, T_{e}\right)=b\left(\frac{Z}{V}\right)^{\beta}\left(1+\mu T_{e} V^{\sigma}\right)^{-1},
$$

where $b, \beta, \sigma, \mu$-parameters, determined from shock compression data of metals.

For the free energy of an ideal Fermi gas we use approximation:

$$
F_{e}=y T_{e}\left[\frac{3}{5} \varphi-\frac{3}{2} \ln \left(1+\frac{5}{2 \varphi}\right)\right],
$$

where

$$
\varphi=\frac{1}{2}\left(3 \pi^{2}\right)^{\frac{2}{3}}\left(\frac{y}{V}\right)^{\frac{2}{3}} / T_{e}
$$


From (10) we get formulas for the chemical potential, pressure and internal energy of the free electrons:

$$
\begin{gathered}
\mu_{F}=\frac{1}{2}\left(3 \pi^{2}\right)^{\frac{2}{3}}\left(\frac{y}{V}\right)^{\frac{2}{3}}+T_{e}\left[\left(1+\frac{2}{5} \varphi\right)^{-1}-\frac{3}{2} \ln \left(1+\frac{5}{2 \varphi}\right)\right], \\
P_{e}=\frac{1}{5}\left(3 \pi^{2}\right)^{\frac{2}{3}}\left(\frac{y}{V}\right)^{\frac{5}{3}}+T_{e}\left(\frac{y}{V}\right)\left(1+\frac{2}{5} \varphi\right)^{-1}, \\
E_{e}=\frac{3}{2} V P .
\end{gathered}
$$

$F_{i}$ is describing the transition from the crystal state at low temperatures to the state of an ideal ion gas at high temperatures:

$$
F_{i}=\frac{3}{2} T_{i} \ln \frac{1+\lambda G}{V^{\frac{2}{3}} T}
$$

where

$$
G=\left(\frac{4 \pi}{3}\right)^{\frac{1}{3}} \frac{y_{c}^{2}(V)}{V^{\frac{1}{3}} T_{i}}
$$

and $y_{c}(V)$ assumed to be a known function of the atomic volume $\mathrm{V}$, defined by the equation of ionization, $\lambda$ - parameter of interpolation.

From (15) we get:

$$
\begin{gathered}
P_{i}=\frac{T_{i}}{V} \frac{\left.1+3 \lambda\left(\frac{1}{2}-\frac{\left.d \ln y_{c}(V)\right)}{d \ln V}\right) G\right)}{1+\lambda G}, \\
E_{i}=\frac{3}{2} T_{i} \frac{1+2 \lambda G}{1+\lambda G} .
\end{gathered}
$$

And finally, for $P_{e i}$ we take into account that it must not affect the equation of ionization, full pressure should be equal zero at normal density and zero temperature, component of the internal energy must take into account the Coulomb attraction between the free electrons, at low densities $P_{e i}$ must decrease rapidly, electronion interaction at a fixed density should decrease as the temperature increases.

$$
P_{e i}=-\frac{1}{5}\left(3 \pi^{2}\right)^{\frac{2}{3}}\left(\frac{y_{c}\left(V_{0}\right)}{V_{0}}\right)^{\frac{5}{3}}\left(\frac{V_{0}}{V}\right)^{\frac{4}{3}} \frac{1+\delta}{1+\left[\delta+(1+\delta) T_{e} / T^{*}\right]\left(\frac{V}{V_{0}}\right)^{\gamma}} .
$$

Here $V_{0}, \gamma, \delta, T^{*}$ are the parameters, determined from the known values of density, sublimation energy, isothermal compressibility and thermal expansion coefficient.

\section{Results}

To verify the model we determine the dependence of ablation depth on the laser pulse duration after a single laser shot and compare with experimental data for 
aluminium and copper. Ultrashort laser pulses are generated by an amplified all solid-state Ti:Sapphire laser chain. ${ }^{8}$

Low energy pulses are extracted from a mode-locked oscillator (1.6 nJ/pulse, 80 $\mathrm{MHz}, 800 \mathrm{~nm}, 120 \mathrm{fs}$ ). The pulses are then injected into an amplifying chain including: an optical pulse stretcher, a regenerative amplifier associated with a two-pass amplifier using a $20 \mathrm{~W}$ Nd:YLF laser as pumping source, and a pulse compressor. Linearly polarized pulses with wavelength centered around $800 \mathrm{~nm}$, an energy of $1.5 \mathrm{~mJ}$ at $1 \mathrm{kHz}$ repetition rate and typical duration of $170 \mathrm{fs}$ were delivered.

The samples are mounted on a three-motorized-axes system with $0.5 \mu \mathrm{m}$ accuracy. Experiments are performed in the image plane of an aperture placed before the objective. The resulting spatial laser profile on sample is 'top hat' so that borders and spurious conical drilling effects are reduced. Focusing objectives of $25 \mathrm{~mm}$ or $10 \mathrm{~mm}$ focal lengths to obtain fluences in a range of 0.5 to $35 \mathrm{~J} / \mathrm{cm}^{2}$ with the same beam size, $16 \mu \mathrm{m}$ in diameter, in the image plane.

As we can see in Fig. 1 and in Fig. 2 results of the simulation are pretty close to experimental data both for copper and aluminium in a range of 0.5 to $35 \mathrm{~J} / \mathrm{cm}^{2}$.

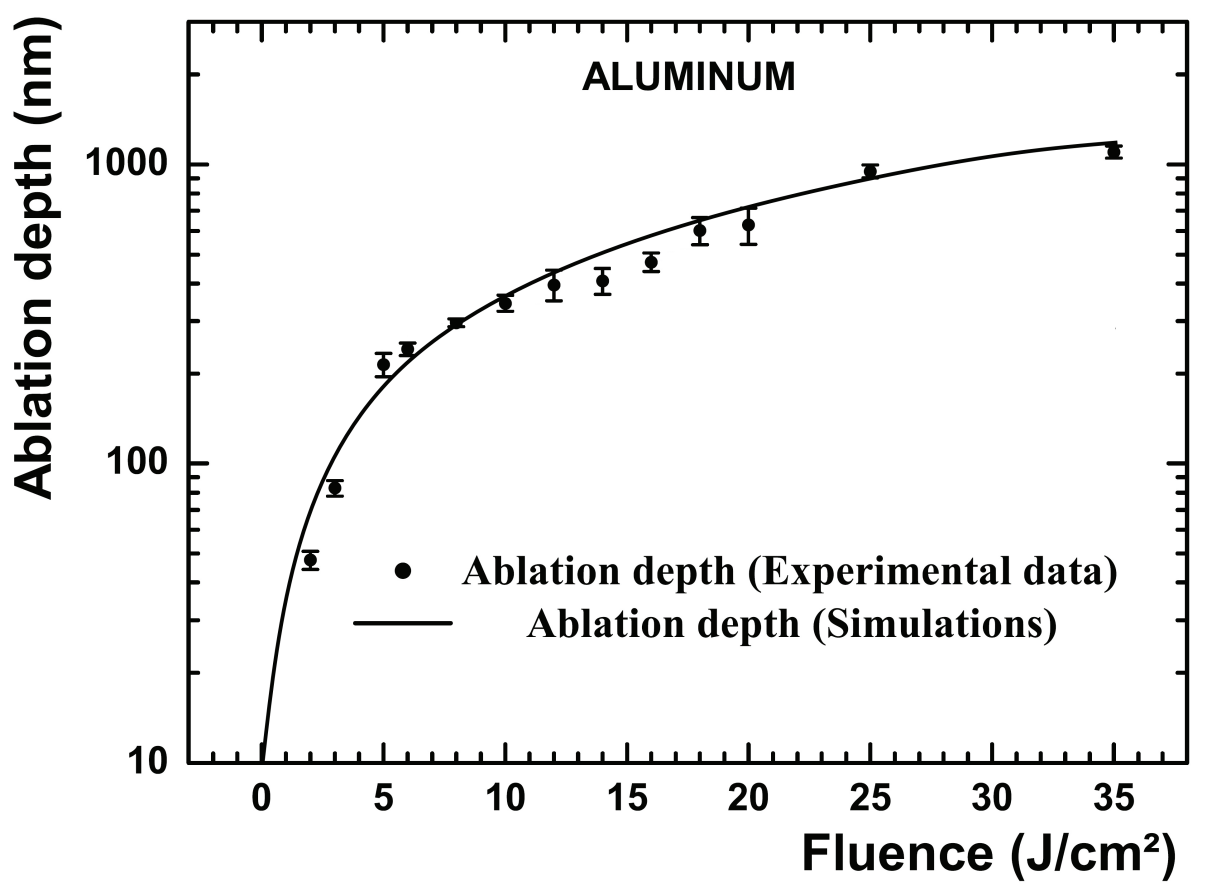

Fig. 1. Experimental and numerical ablation depth as a function of the laser fluence on an aluminum target obtained with a 170 fs laser pulse. 


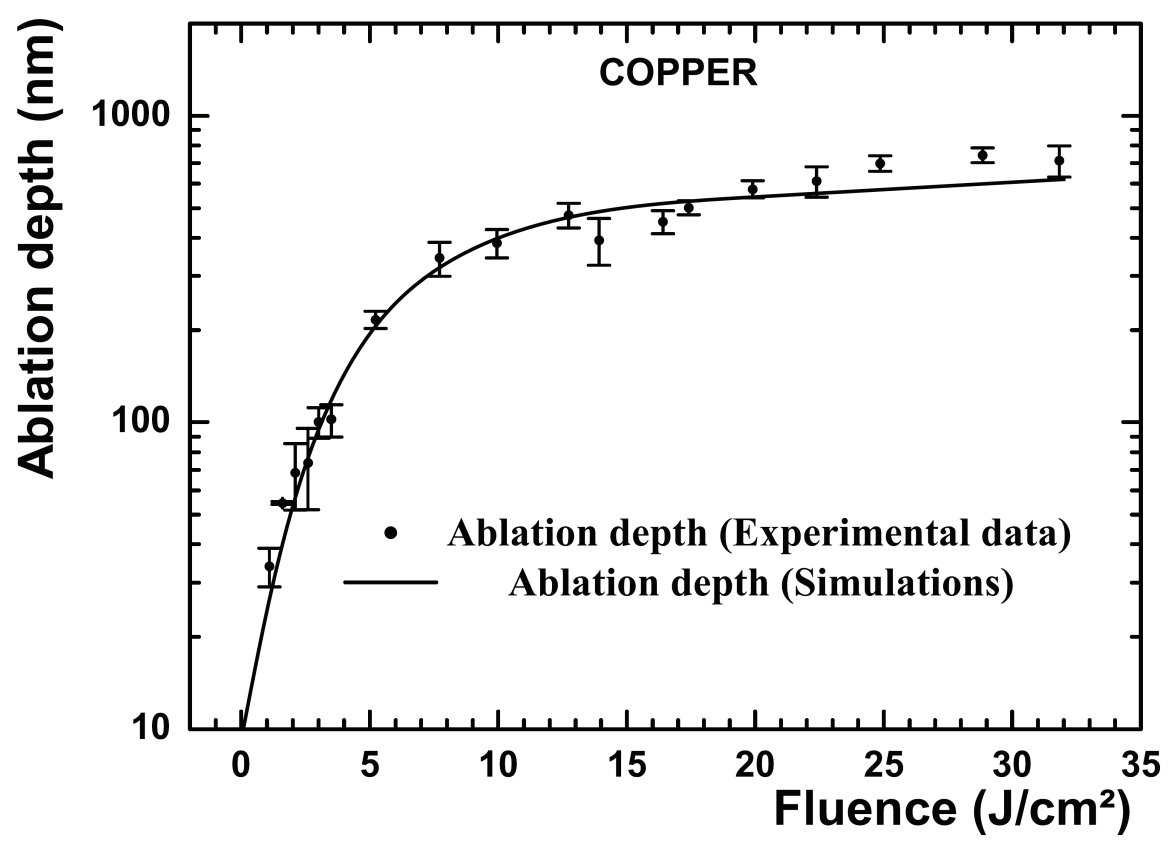

Fig. 2. Experimental and numerical ablation depth as a function of the laser fluence on a copper target obtained with a 170 fs laser pulse.

\section{Conclusion}

In this paper a mathematical model for femtosecond laser ablation of metals is proposed, based on standard two-temperature model connected with 1D hydrodynamic equations. Wide-range equation of state has been developed. The simulation results are compared with experimental data for aluminium and copper. A good agreement for both metals with numerical results and experiment shows that this model can be employed for choosing laser parameters to better accuracy in nanoparticles production by ablation of metals.

\section{References}

1. G. N. Makarov, Phys. Usp. 56, 643 (2013).

2. Q. Li, H. Lao, J. Lin, Y. Chen and X. Chen, Appl. Phys. A 105, 125 (2011).

3. Y. Ren, J. Chen and Y. Zhang, Int. J. Heat Mass Transfer 55, 1620 (2012).

4. S. Mishra and V. Yadava, Opt. Laser Technol. 48, 461 (2013).

5. J. Zhang, Y. Chen, M. Hu and X. Chen, J. Appl. Phys. 117, 063104 (2015).

6. Yu. V. Petrov, J. Laser and Particle Beams 23, 283 (2005).

7. M. E. Povarnitsyn, N. E. Andreev, P. R. Levashov, K. V. Khishchenko and O. N. Rosmej, Phys. Plasmas 19, 023110 (2012).

8. J. P. Colombier, P. Combis, F. Bonneau, R. Le Harzic and E. Audouard, Phys. Rev. B 71, 165406 (2005). 